J. Korean Math. Soc. 49 (2012), No. 3, pp. 503-514

http://dx.doi.org/10.4134/JKMS.2012.49.3.503

\title{
GENERALIZATIONS OF T-EXTENDING MODULES RELATIVE TO FULLY INVARIANT SUBMODULES
}

\author{
Shadi Asgari And Ahmad Haghany
}

\begin{abstract}
The concepts of t-extending and t-Baer for modules are generalized to those of FI-t-extending and FI-t-Baer respectively. These are also generalizations of FI-extending and nonsingular quasi-Baer properties respectively and they are inherited by direct summands. We shall establish a close connection between the properties of FI-t-extending and FI-t-Baer, and give a characterization of FI-t-extending modules relative to an annihilator condition.
\end{abstract}

\section{Introduction}

Recall that a submodule $K$ of an $R$-module $M$ is called fully invariant if $\varphi(K) \leq K$ for every $R$-endomorphism $\varphi$ of $M$. For example, the Jacobson radical, the socle, the singular submodule $Z(M)$, the torsion submodule or second singular submodule $Z_{2}(M)$ and the submodules $M I$ for every right ideal $I$ of $R$ are fully invariant in $M$. A module $M$ is called FI-extending if every fully invariant submodule of $M$ is essential in a direct summand of $M$. FI-extending modules were introduced in [3] and further studied in [2], [4], [5], and [6]. In [1] we called a submodule $A$ of $M$ t-essential in $M$ (written $A \leq_{\text {tes }} M$ ) if for every submodule $B$ of $M, A \cap B \leq Z_{2}(M)$ implies that $B \leq Z_{2}(M)$. Indeed a t-essential submodule of $M$ is a dense submodule of $M$ in the Goldie torsion theory on Mod- $R$ and so the notion of a t-essential submodule is a generalization of that of an essential submodule. A submodule $C$ of $M$ is called t-closed in $M$ (written $C \leq_{t c} M$ ) if $C \leq_{t e s} C^{\prime} \leq M$ implies that $C=C^{\prime}$. As in [1], a module $M$ is called t-extending if every t-closed submodule of $M$ is a direct summand. Indeed, $M$ is t-extending if and only if every submodule of $M$ is t-essential in a direct summand [1, Theorem 2.11]. Now it is natural to ask: When does a module have the property that every fully invariant submodule is t-essential in a direct summand? In [4] a module $M$ is called strongly FI-extending if every fully invariant submodule is essential in a fully invariant direct summand. This class of modules is properly contained in the class of

Received June 6, 2010.

2010 Mathematics Subject Classification. 16D10, 16D80, 16D70.

Key words and phrases. nonsingular and $Z_{2}$-torsion modules, t-closed submodules, FIextending and FI-t-extending modules, quasi-Baer and FI-t-Baer modules. 
FI-extending modules. Again it is natural to ask: When does a module have the property that every fully invariant submodule is t-essential in a fully invariant direct summand?

The main purpose of this paper is to answer these questions. We say a module $M$ is FI-t-extending if every fully invariant t-closed submodule of $M$ is a direct summand of $M$. FI-extending modules, t-extending modules (hence extending modules, all finitely generated abelian groups) and projective modules over a ring $R$ for which $R_{R}$ is FI-extending or t-extending, are examples of FIt-extending modules. We will show in Theorem 2.2 that every fully invariant submodule of a module $M$ is t-essential in a direct summand if and only if every fully invariant submodule of $M$ is t-essential in a fully invariant direct summand and that these are equivalent to $M$ being FI-t-extending. In addition, we show that an FI-t-extending module is exactly a direct sum of a nonsingular FI-extending module and a $Z_{2}$-torsion module. By a $Z_{2}$-torsion module $K$ we mean any module $K$ with $Z_{2}(K)=K$. Similar to the FI-extending modules, every direct sum of FI-t-extending modules is FI-t-extending and every fully invariant submodule of any FI-t-extending module inherits the property. Although it is not known whether a direct summand of an FI-extending module is FI-extending, we will see that a direct summand of an FI-t-extending module inherits the property (Corollary 2.4). As a consequence, a direct summand $N$ of an FI-extending module is FI-extending if and only if $Z_{2}(N)$ is FI-extending. In particular every direct summand of an FI-extending module $M$ is FI-extending if $Z_{2}(M)$ is extending, strongly FI-extending or weak duo.

For a left ideal $I$ of $\operatorname{End}(M)$, set $r_{M}(I)=\{m \in M: I m=0\}$ and $t_{M}(I)=\left\{m \in M: I m \leq Z_{2}(M)\right\}$. Recall from [10] that a module $M$ is (quasi-)Baer if the right annihilator in $M$ of any (two-sided) left ideal $I$ of $\operatorname{End}(M)$ (i.e., $\left.r_{M}(I)\right)$ is a direct summand of $M$. The notion of a (quasi-)Baer module $M$ coincides with that of a (quasi-)Baer ring when $M=R_{R}$. A close connection was established between (quasi-)Baer modules and (FI-) extending modules in [10, Theorems 2.12 and 3.10]. In [1] we have introduced the notion of a t-Baer module which is a generalization of the notions of a t-extending module (hence an extending module) and of a nonsingular Baer module. In fact, a module $M$ is t-Baer if $t_{M}(I)$ is a direct summand of $M$ for any left ideal $I$ of $\operatorname{End}(M)$. There is a connection between t-extending and t-Baer properties, that is, a module $M$ is t-extending if and only if it is t-Baer and t-cononsingular [1, Theorem 3.9]. We say that a module $M$ is FI-t-Baer if $t_{M}(I)$ is a direct summand of $M$ for any two-sided ideal $I$ of $\operatorname{End}(M)$. Every t-Baer module and every nonsingular quasi-Baer module is FI-t-Baer. We give some equivalent conditions to being FI-t-Baer similar to [1, Theorem 3.2] which is for a t-Baer module. Moreover we show that a module $M$ is FI-t-extending if and only if it is FI-t-Baer and FI-t-cononsingular (Theorem 3.9).

A characterization of a quasi-continuous module relative to an annihilator condition is given in [11, Theorem 8], which states that $M$ is quasi-continuous if and only if $S=l_{S}(A)+l_{S}(B)$ for any submodules $A$ and $B$ of $M$ with 
$A \cap B=0$ if and only if $S=l_{S}(A)+l_{S}(B)$ (or equivalently, $S=l_{S}(A) \oplus l_{S}(B)$ ) for any submodules $A$ and $B$ of $M$ which are complements to each other, where $S=\operatorname{End}(M)$ and $l_{S}(A)$ and $l_{S}(B)$ are annihilators of $A$ and $B$ in $S$ respectively. Analogous to this, in [7, Corollary 2.5], it is shown that a module $M$ is extending if and only if for every closed submodule $C$ of $M$ there exists a complement $D$ of $C$ in $M$ such that $S=l_{S}(C)+l_{S}(D)$ (or equivalently, $\left.S=l_{S}(C) \oplus l_{S}(D)\right)$. We will show in Theorem 4.1 that there is a similar characterization for FI-t-extending modules. In fact, a module $M$ is FI-t-extending if and only if for every fully invariant t-closed submodule $C$ of $M$ there exists a complement $D$ of $C$ in $M$ such that $S=l_{S}(C)+l_{S}(D)$ (or equivalently, $\left.S=l_{S}(C) \oplus l_{S}(D)\right)$ if and only if for every fully invariant t-closed submodule $C$ of $M$ there exists a complement $D$ to $C$ in $M$ such that $D+Z_{2}(M)$ is t-closed in $M$ and $S=t_{S}(C)+t_{S}(D)$, where $t_{S}(N)=\left\{\varphi \in S: \varphi N \leq Z_{2}(M)\right\}$ for a submodule $N$ of $M$.

We end this section by recording the following facts for future use.

Proposition 1.1 ([1, Proposition 2.2]). The following statements are equivalent for a submodule $A$ of an $R$-module $M$.

(1) $A$ is t-essential in $M$.

(2) $\left(A+Z_{2}(M)\right) / Z_{2}(M)$ is essential in $M / Z_{2}(M)$.

(3) $A+Z_{2}(M)$ is essential in $M$.

(4) $M / A$ is $Z_{2}$-torsion.

Proposition 1.2 ([1, Proposition 2.6]). Let $C$ be a submodule of a module $M$. The following statements are equivalent.

(1) $C$ is t-closed in $M$.

(2) $C$ contains $Z_{2}(M)$ and $C$ is a closed submodule of $M$.

(3) $M / C$ is nonsingular.

Proposition 1.3. Let $K \leq N$ be submodules of a module $M$. If $K$ is fully invariant in $M$ and $N / K$ is fully invariant in $M / K$, then $N$ is fully invariant in $M$.

Proof. This is routine.

\section{FI-t-extending modules}

Throughout rings will have unity and modules will be unitary. Unless stated otherwise, modules will be right modules. Recall from [1] that a module $M$ is t-extending if every t-closed submodule is a direct summand. By restricting to fully invariant t-closed submodules of $M$ we have the following notion.

Definition 2.1. We say that a module $M$ is FI-t-extending if every fully invariant t-closed submodule of $M$ is a direct summand of $M$.

Clearly every t-extending module is FI-t-extending. From Theorem 2.2(8) below, we conclude that every FI-extending module is FI-t-extending. The 
properties of strongly FI-extending, FI-extending and FI-t-extending are identified for a nonsingular module; see [4, Proposition 1.5].

Theorem 2.2. The following statements are equivalent for a module $M$.

(1) $M$ is FI-t-extending.

(2) For every fully invariant submodule $A$ of $M, A_{2}$ is a direct summand of $M$ where $A_{2} / A=Z_{2}(M / A)$.

(3) $M=Z_{2}(M) \bigoplus M^{\prime}$ where $M^{\prime}$ is a (nonsingular) FI-extending module.

(4) Every fully invariant submodule of $M$ which contains $Z_{2}(M)$ is essential in a direct summand of $M$.

(5) Every essential closure of a fully invariant submodule of $M$ which contains $Z_{2}(M)$ is a direct summand of $M$.

(6) Every fully invariant submodule of $M$ which contains $Z_{2}(M)$ is essential in a fully invariant direct summand of $M$.

(7) Every fully invariant submodule of $M$ is t-essential in a fully invariant direct summand.

(8) Every fully invariant submodule of $M$ is t-essential in a direct summand.

(9) For every fully invariant submodule $A$ of $M$, there exists a decomposition $M / A=N / A \bigoplus N^{\prime} / A$ such that $N$ is a direct summand of $M$ and $N^{\prime} \leq_{\text {tes }} M$.

Proof. For $(1) \Rightarrow(2) \Rightarrow(3) \Rightarrow(4)$ and $(8) \Rightarrow(9) \Rightarrow(1)$ follow the proof of $(1) \Rightarrow(2) \Rightarrow(3) \Rightarrow(4)$ and $(5) \Rightarrow(6) \Rightarrow(1)$ of [1, Theorem 2.11] respectively, by assuming there, that $C, K$ and $A$ are fully invariant. Note that in $(2) \Rightarrow(3)$, it is enough to show that $C$ is a direct summand by [4, Proposition 1.5]. The implication $(7) \Rightarrow(8)$ is clear.

$(4) \Rightarrow(5)$. Let $A$ be a fully invariant submodule of $M$ which contains $Z_{2}(M)$ and $\bar{A}$ be an essential closure of $A$ (that is, $\bar{A}$ is a maximal member of the set of submodules of $M$ which $A$ is essential in them). Clearly $\bar{A}$ is a closed submodule which contains $Z_{2}(M)$, hence by Proposition $1.2, \bar{A}$ is t-closed. Now we show that $\bar{A}$ is fully invariant. Assume that $\varphi$ is an endomorphism of $M$ and $x \in \bar{A}$. There exists an essential right ideal $I$ such that $x I \leq A$, hence $\varphi(x) I=\varphi(x I) \leq A$. Thus $\varphi(x)+\bar{A} \in Z(M / \bar{A})$ and so by Proposition 1.2(3), $\varphi(x) \in \bar{A}$. Therefore $\bar{A}$ is fully invariant, hence is essential in a direct summand by (4), thus it is a direct summand of $M$.

$(5) \Rightarrow(6)$. Let $A$ be a fully invariant submodule of $M$ which contains $Z_{2}(M)$. As shown in the previous part, an essential closure of $A$ is fully invariant and so it serves as such a desired direct summand.

$(6) \Rightarrow(7)$. Let $A$ be a fully invariant submodule of $M$. Clearly $A+Z_{2}(M)$ is also fully invariant, hence there exists a fully invariant direct summand $N$ of $M$ such that $A+Z_{2}(M)$ is essential in $N$. Thus by Proposition $1.1, A \leq_{\text {tes }} N$.

Corollary 2.3. Every direct sum of FI-t-extending modules is FI-t-extending.

Proof. This is clear by Theorem 2.2(3) and [3, Theorem 1.3].

Corollary 2.4. Let $M$ be an FI-t-extending module. 
(1) $M / K$ is FI-t-extending for every fully invariant submodule $K$ of $M$.

(2) Every fully invariant submodule of $M$ is FI-t-extending.

(3) Every direct summand of $M$ is FI-t-extending.

Proof. (1) Follow the proof of [1, Proposition 2.14(1)] by assuming there, that $K$ is a fully invariant submodule of $M$ and $L / K$ is a fully invariant submodule of $M / K$ and then apply Proposition 1.3.

(2) Follow the proof of [1, Proposition 2.14(2)] by assuming there, that $L$ is a fully invariant submodule of $M$ and $K$ is a fully invariant submodule of $L$.

(3) Let $N$ be a direct summand of $M$, say $M=N \oplus N^{\prime}$. First assume that $N$ is nonsingular. By Theorem 2.2(3), $M=Z_{2}(M) \oplus M^{\prime}$ and so $N^{\prime}=$ $Z_{2}(M) \oplus\left(N^{\prime} \cap M^{\prime}\right)$. Hence $M=N \oplus\left(N^{\prime} \cap M^{\prime}\right) \oplus Z_{2}(M)$. Therefore by (1), $N \oplus\left(N^{\prime} \cap M^{\prime}\right)$ is strongly FI-extending and so by [4, Theorem 2.4], $N$ is strongly FI-extending, hence it is FI-t-extending.

Now if $N$ is not nonsingular, then $Z_{2}(M)=Z_{2}(N) \oplus Z_{2}\left(N^{\prime}\right)$ and so by Theorem 2.2(3), $N=Z_{2}(N) \oplus L$ for some submodule $L$. However $L$ is a nonsingular direct summand of $M$, hence by what we showed first $L$ is strongly FI-extending. Thus $N$ is FI-t-extending.

Corollary 2.5. Let $R$ be a ring. Then $R_{R}$ is FI-t-extending if and only if every projective $R$-module is FI-t-extending.

Corollary 2.6. The following are equivalent for a module $M$.

(1) $M$ is FI-extending.

(2) $M=Z_{2}(M) \bigoplus M^{\prime}$ where $Z_{2}(M)$ and $M^{\prime}$ are FI-extending.

Proof. (1) $\Rightarrow(2)$. By Theorem 2.2(3), $M=Z_{2}(M) \oplus M^{\prime}$ where $M^{\prime}$ is FIextending. Thus it suffices to show that $Z_{2}(M)$ is FI-extending. Let $A$ be a fully invariant submodule of $Z_{2}(M)$. Since $Z_{2}(M)$ is a fully invariant submodule of $M, A$ is a fully invariant submodule of $M$. Therefore $A$ is essential in a direct summand $N$ of $M$, say $M=N \oplus N^{\prime}$. However $A$ and $N / A$ are $Z_{2}$-torsion, hence $N$ is $Z_{2}$-torsion and so $N \leq Z_{2}(M)$. Thus $Z_{2}(M)=N \bigoplus\left(Z_{2}(M) \cap N^{\prime}\right)$, hence $N$ is a direct summand of $Z_{2}(M)$. This implies that $Z_{2}(M)$ is FI-extending.

$(2) \Rightarrow(1)$. This follows from the fact that a direct sum of FI-extending modules is FI-extending [3, Theorem 1.3].

Remark 2.7. The implication (1) $\Rightarrow(2)$ of Corollary 2.6 can also be obtained from [4, Proposition 2.8 and Proposition 1.5].

Corollary 2.8. A module $M$ is FI-extending if and only if $M$ is FI-t-extending and $Z_{2}(M)$ is FI-extending.

Proof. This is clear by Corollary 2.6, Theorem 2.2 and [3, Theorem 1.3].

Recall from [9] that a module $M$ is (weak) duo if every (direct summand) submodule of $M$ is fully invariant. In [3] there is an open problem asking whether a direct summand of an FI-extending module is FI-extending. Clearly 
this is true if the FI-extending module is weak duo. The next corollary, in particular, shows that the above problem has an affirmative answer when $Z_{2}(M)$ is weak duo. In fact this gives a necessary and sufficient condition for a direct summand of an FI-extending module to be FI-extending.

Corollary 2.9. Let $M$ be an FI-extending module.

(1) If $N$ is a direct summand of $M$, then $N$ is FI-extending if and only if $Z_{2}(N)$ is FI-extending.

(2) Every direct summand of $M$ is FI-extending if and only if every direct summand of $Z_{2}(M)$ is FI-extending. In particular, if $Z_{2}(M)$ is weak duo, extending or strongly FI-extending, then every direct summand of $M$ is FIextending.

Proof. (1) is obtained by Corollaries 2.6, 2.4(3) and 2.8, while (2) follows from (1).

The next examples shows that the class of FI-t-extending modules properly contains both the class of t-extending modules and the class of FI-extending modules.

Examples 2.10. (1) Let $R=\left(\begin{array}{c}\mathbb{Z} \\ 0\end{array}\right)$ and $M$ be an arbitrary $R$-module. Then $R_{R}$ is FI-extending, but it is not extending; see [3, Example 2.6]. Note that $R$ is right nonsingular and so by Theorem 2.2(3) and [1, Theorem 2.11(3)], $R \oplus Z_{2}(M)$ is an FI-t-extending $R$-module which is not t-extending.

(2) A characterization of an FI-extending $Z_{2}$-torsion group is given in $[2$, Theorem 2.3]. So every $Z_{2}$-torsion $\mathbb{Z}$-module which is not FI-extending is an example of an FI-t-extending module which is not FI-extending.

\section{FI-t-Baer modules}

Let $S=\operatorname{End}(M)$ and $I$ be a left ideal of $S$. Set $r_{M}(I)=\{m \in M: I m=0\}$ and $t_{M}(I)=\left\{m \in M: I m \leq Z_{2}(M)\right\}$. In addition, for a submodule $N$ of $M$, set $l_{S}(N)=\{\varphi \in S: \varphi N=0\}$ and $t_{S}(N)=\left\{\varphi \in S: \varphi N \leq Z_{2}(M)\right\}$. Recall from [10] that a module $M$ is quasi-Baer if for every fully invariant submodule $N$ of $M$, the two-sided ideal $l_{S}(N)$ is a direct summand of $S$ as a left ideal; equivalently, for every two-sided ideal $J$ of $S$, the submodule $r_{M}(J)$ is a direct summand of $M$. Moreover, recall from [1] that a module $M$ is t-Baer if $t_{M}(I)$ is a direct summand of $M$ for every left ideal $I$ of $S$. By restricting the t-Baer requirement to the two-sided ideals of $S$ we have the following notion.

Definition 3.1. A module $M$ is FI-t-Baer if $t_{M}(J)$ is a direct summand of $M$ for every two-sided ideal $J$ of $S$.

Clearly every t-Baer module is FI-t-Baer, and the properties of FI-t-Baer and quasi-Baer coincide for a nonsingular module.

Analogous to [1, Theorem 3.9], there is a connection between FI-t-extending 
modules and FI-t-Baer modules. Before establishing this, we give some characterizations of FI-t-Baer modules which are analogous to the characterizations of t-Baer modules [1, Theorem 3.2].

Theorem 3.2. The following statements are equivalent for a module $M$.

(1) $M$ is FI-t-Baer.

(2) $M=Z_{2}(M) \bigoplus M^{\prime}$ where $M^{\prime}$ is a (nonsingular) quasi-Baer module.

(3) $M$ has the strong summand intersection property for fully invariant direct summands which contain $Z_{2}(M)$, and $t_{M}(J)$ is a direct summand of $M$ for all principal two-sided ideals $J$ of $S$.

(4) $\bigcap_{\varphi \in \mathcal{T}} t_{M}(S \varphi S)$ is a direct summand of $M$ for every subset $\mathcal{T}$ of $S$.

Proof. $(1) \Rightarrow(2)$. Since $M$ is FI-t-Baer, $Z_{2}(M)=t_{M}(S)$ is a direct summand of $M$, say $M=Z_{2}(M) \oplus M^{\prime}$. Now we show that $M^{\prime}$ is quasi-Baer. Let $J^{\prime}$ be a two-sided ideal of $S^{\prime}=\operatorname{End}\left(\mathrm{M}^{\prime}\right), A=\left\{1 \oplus \psi: \psi \in J^{\prime}\right\}$ and $J=S A S$. So $t_{M}(J)=Z_{2}(M) \bigoplus r_{M^{\prime}}\left(J^{\prime}\right)$. Since $M$ is FI-t-Baer, $t_{M}(J)$ is a direct summand of $M$ and so $r_{M^{\prime}}\left(J^{\prime}\right)$ is a direct summand of $M^{\prime}$. Thus $M^{\prime}$ is a quasi-Baer module.

$(2) \Rightarrow(1)$. Assume that $M=Z_{2}(M) \bigoplus M^{\prime}$ where $M^{\prime}$ is a quasi-Baer module. Let $S^{\prime}=\operatorname{End}\left(\mathrm{M}^{\prime}\right), J$ be a two-sided ideal of $S, A^{\prime}=\left\{\left.\pi^{\prime} \varphi\right|_{M^{\prime}}: \varphi \in J\right\}$ where $\pi^{\prime}$ is the canonical projection to $M^{\prime}$, and $J^{\prime}=S^{\prime} A^{\prime} S^{\prime}$. Thus $t_{M}(J)=$ $Z_{2}(M) \bigoplus r_{M^{\prime}}\left(J^{\prime}\right)$. Since $M^{\prime}$ is quasi-Baer, $r_{M^{\prime}}\left(J^{\prime}\right)$ is a direct summand of $M^{\prime}$, hence $t_{M}(J)$ is a direct summand of $M$.

$(1) \Rightarrow(3)$. Assume that $\left\{e_{\lambda}: \lambda \in \Lambda\right\}$ is a set of idempotents of $S$ such that $e_{\lambda} M$ contains $Z_{2}(M)$ and is fully invariant submodule of $M$. Let $J=\sum_{\lambda \in \Lambda} S\left(1-e_{\lambda}\right) S$. Then $J$ is a two-sided ideal of $S$ with $t_{M}(J) \leq$ $\left(1-e_{\lambda}\right)^{-1} Z_{2}(M)=e_{\lambda} M$ for each $\lambda \in \Lambda$, and so $t_{M}(J) \leq \bigcap_{\lambda \in \Lambda} e_{\lambda} M$. If $m \notin t_{M}(J)$, there exist $\lambda_{0} \in \Lambda$ and $\theta \in S$ such that $\left(1-e_{\lambda_{0}}\right) \theta m \notin Z_{2}(M)$, hence $\theta m \notin e_{\lambda_{0}} M=\left(1-e_{\lambda_{0}}\right)^{-1} Z_{2}(M)$. Since $e_{\lambda_{0}} M$ is fully invariant, we conclude that $m \notin e_{\lambda_{0}} M$. Thus $m \notin \bigcap_{\lambda \in \Lambda} e_{\lambda} M$ and so $\bigcap_{\lambda \in \Lambda} e_{\lambda} M=t_{M}(J)$, hence $\bigcap_{\lambda \in \Lambda} e_{\lambda} M$ is a direct summand of $M$ as $M$ is FI-t-Baer. The second statement is clear.

$(3) \Rightarrow(4)$. Since $S \varphi S$ is a two-sided ideal of $S, t_{M}(S \varphi S)$ is a fully invariant submodule of $M$, and $t_{M}(S \varphi S)$ contains $Z_{2}(M)$ for every $\varphi \in S$, the implication is clear.

$(4) \Rightarrow(1)$. Let $J$ be a two-sided ideal of $S$. Clearly $t_{M}(J)=\bigcap_{\varphi \in J} t_{M}(S \varphi S)$. Thus by assumption $t_{M}(J)$ is a direct summand of $M$ and so $M$ is FI-tBaer.

Corollary 3.3. Let $M=\bigoplus_{\lambda \in \Lambda} M_{\lambda}$ such that each $M_{\lambda}$ is FI-t-Baer and subisomorphic to $M_{\mu}$ for all $\mu \in \Lambda$. Then $M$ is FI-t-Baer.

Proof. Clearly $M_{\lambda} / Z_{2}\left(M_{\lambda}\right)$ is subisomorphic to $M_{\mu} / Z_{2}\left(M_{\mu}\right)$ for all $\lambda, \mu \in \Lambda$. Thus the result follows by Theorem 3.2(2) and [10, Proposition 3.19]. 
Examples 3.4. (1) Let $R$ be a Baer ring. Then by Theorem 3.2 and [10, Corollary 3.20 and Theorem 3.17], $P \oplus Z_{2}(M)$ is FI-t-Baer for every projective $R$-module $P$ and every $R$-module $M$.

(2) It is well-known that the upper triangular matrix ring over a domain which is not a division ring is quasi-Baer but not Baer. Therefore by Examples 2.10(1), Theorem 3.2 and [1, Theorem 3.2], there exist modules which are FIt-Baer but not t-Baer. Hence the class of FI-t-Baer modules properly contains the class of t-Baer modules.

Proposition 3.5. If $M$ is FI-t-Baer, then so is every direct summand of $M$.

Proof. First assume that $M=M_{1} \bigoplus M_{2}$ is FI-t-Baer and $M_{1}$ is $Z_{2}$-torsion. Then $M_{2}$ is FI-t-Baer; in fact if $I_{2}$ is a two-sided ideal of $S_{2}=\operatorname{End}\left(M_{2}\right)$, $A=\left\{1_{M_{1}} \oplus \varphi: \varphi \in I_{2}\right\}$ and $I=S A S$, then $t_{M}(I)=M_{1} \oplus t_{M_{2}}\left(I_{2}\right)$. By hypothesis $t_{M}(I)$ is a direct summand of $M$, hence $t_{M_{2}}\left(I_{2}\right)$ is a direct summand of $M_{2}$.

Now let $N$ be a direct summand of $M$, say $M=K \bigoplus N$. Since $M$ is FI-tBaer, $Z_{2}(M)=Z_{2}(K) \oplus Z_{2}(N)$ is a direct summand of $M$. Hence $Z_{2}(K)$ is a direct summand of $K$. Set $L=Z_{2}(K) \bigoplus N$. Then $L$ is a direct summand of $M$ which contains $Z_{2}(M)$. By the first paragraph it suffices to show that $L$ is FI-tBaer. Since $M=Z_{2}(M) \bigoplus M^{\prime}$ where $M^{\prime}$ is quasi-Baer, $L=Z_{2}(M) \bigoplus\left(L \cap M^{\prime}\right)$. Thus $L \cap M^{\prime}$ is a direct summand of $M$, hence it is a direct summand of $M^{\prime}$. Therefore $L \cap M^{\prime}$ is quasi-Baer by [10, Theorem 3.17] and so $L$ is FI-t-Baer, as desired.

Corollary 3.6. Let $R$ be a ring. Then $R_{R}$ is FI-t-Baer if and only if every projective $R$-module is FI-t-Baer.

Proof. This follows by Corollary 3.3 and Proposition 3.5.

In [10] a module $M$ is called FI- $\mathcal{K}$-cononsingular if for every fully invariant direct summand $N$ of $M$ and every fully invariant submodule $K$ of $N, l_{S^{\prime}}(K)=$ 0 implies that $K$ is essential in $N$, where $S^{\prime}=\operatorname{End}(N)$.

Definition 3.7. We say that a module $M$ is FI-t-cononsingular if for every fully invariant submodule $N$ of $M$ and every fully invariant submodule $K$ of $N, t_{S^{\prime}}(K)=t_{S^{\prime}}(N)$ implies that $K$ is t-essential in $N$, where $S^{\prime}=\operatorname{End}(N)$.

Clearly, every $Z_{2}$-torsion and every nonsingular uniform module is FI-tcononsingular.

Proposition 3.8. Let $M$ be a module.

(1) If $M$ is FI-t-cononsingular, then $M / Z_{2}(M)$ is FI-K-cononsingular.

(2) If $M=\bigoplus_{\lambda \in \Lambda} M_{\lambda}$ where each $M_{\lambda}$ is FI-t-cononsingular, then $M$ is FIt-cononsingular.

(3) If $M=M_{1} \bigoplus M_{2}$ is FI-t-cononsingular and $M_{1}$ is $Z_{2}$-torsion, then $M_{2}$ is FI-t-cononsingular. 
Proof. (1) Let $N / Z_{2}(M)$ be a fully invariant direct summand of $M / Z_{2}(M)$. Set $S^{\prime}=\operatorname{End}(N)$ and $\bar{S}=\operatorname{End}\left(N / Z_{2}(M)\right)$. Assume that $K / Z_{2}(M)$ is a fully invariant submodule of $N / Z_{2}(M)$ such that $l_{\bar{S}}\left(K / Z_{2}(M)\right)=0$. Then $t_{S^{\prime}}(K)=$ $t_{S^{\prime}}(N)$; in fact if $\varphi \in t_{S^{\prime}}(K)$, then $\bar{\varphi}: N / Z_{2}(M) \rightarrow N / Z_{2}(M)$ defined by $\varphi(x+$ $\left.Z_{2}(M)\right)=\varphi(x)+Z_{2}(M)$ is an endomorphism of $N / Z_{2}(M)$, and $\bar{\varphi}\left(K / Z_{2}(M)\right)=$ 0 . Thus $\bar{\varphi}=0$ and so $\varphi \in t_{S^{\prime}}(N)$. This implies that $t_{S^{\prime}}(K)=t_{S^{\prime}}(N)$. However by Proposition 1.3, $K$ is a fully invariant submodule of $N$ and $N$ is a fully invariant submodule of $M$, hence by hypothesis $K$ is t-essential in $N$. Thus by Proposition $1.1(2), K / Z_{2}(M)$ is essential in $N / Z_{2}(M)$.

(2) Assume that $N$ is a fully invariant submodule of $M$ and $K$ is a fully invariant submodule of $N$ such that $t_{S^{\prime}}(K)=t_{S^{\prime}}(N)$. Clearly $N=\bigoplus_{\lambda \in \Lambda}(N \cap$ $\left.M_{\lambda}\right)$, each $N \cap M_{\lambda}$ is fully invariant in $M_{\lambda}$, also $K=\bigoplus_{\lambda \in \Lambda}\left(K \cap M_{\lambda}\right)$ and each $K \cap M_{\lambda}$ is fully invariant in $N \cap M_{\lambda}$. Let $S_{\lambda}=\operatorname{End}\left(N \cap M_{\lambda}\right)$. It is easy to see that $t_{S_{\lambda}}\left(K \cap M_{\lambda}\right)=t_{S_{\lambda}}\left(N \cap M_{\lambda}\right)$, hence by assumption $K \cap M_{\lambda} \leq_{\text {tes }} N \cap M_{\lambda}$. Thus by Proposition 1.1(4), $K \leq_{\text {tes }} N$.

(3) Let $N_{2}$ be a fully invariant submodule of $M_{2}$ and $K_{2}$ be a fully invariant submodule of $N_{2}$ such that $t_{S_{2}}\left(K_{2}\right)=t_{S_{2}}\left(N_{2}\right)$ where $S_{2}=\operatorname{End}\left(N_{2}\right)$. By [10, Lemma 1.11], there exists a fully invariant submodule $N_{1}$ of $M_{1}$ such that $N_{1} \oplus N_{2}$ is a fully invariant submodule of $M$. Similarly, there exists a fully invariant submodule $K_{1}$ of $N_{1}$ such that $K_{1} \bigoplus K_{2}$ is a fully invariant submodule of $N_{1} \oplus N_{2}$. So $t_{S^{\prime}}\left(K_{1} \oplus K_{2}\right)=t_{S^{\prime}}\left(N_{1} \oplus N_{2}\right)$ where $S^{\prime}=\operatorname{End}\left(N_{1} \oplus N_{2}\right)$; in fact, if $\varphi \in S^{\prime}$ and $\varphi\left(K_{1} \oplus K_{2}\right) \leq Z_{2}\left(N_{1} \oplus N_{2}\right)$, then $N_{1} \leq Z_{2}\left(N_{1} \oplus N_{2}\right)=$ $N_{1} \oplus Z_{2}\left(N_{2}\right)$ implies that $\pi_{2} \varphi \iota_{2} K_{2} \leq Z_{2}\left(N_{2}\right)$ where $\iota_{2}: N_{2} \rightarrow N_{1} \oplus N_{2}$ and $\pi_{2}: N_{1} \oplus N_{2} \rightarrow N_{2}$ are respectively the canonical injection and projection. Now $\pi_{2} \varphi \iota_{2} \in t_{S_{2}}\left(K_{2}\right)$, hence $\pi_{2} \varphi \iota_{2} \in t_{S_{2}}\left(N_{2}\right)$ and so $\varphi\left(N_{1} \oplus N_{2}\right) \leq Z_{2}\left(N_{1} \oplus N_{2}\right)$. Therefore $\varphi \in t_{S^{\prime}}\left(N_{1} \oplus N_{2}\right)$ and $t_{S^{\prime}}\left(K_{1} \oplus K_{2}\right)=t_{S^{\prime}}\left(N_{1} \oplus N_{2}\right)$, as desired. Since $M$ is FI-t-cononsingular, the latter implies that $K_{1} \bigoplus K_{2} \leq_{\text {tes }} N_{1} \bigoplus N_{2}$ and so $K_{2} \leq_{\text {tes }} N_{2}$ by Proposition 1.1(4).

Next, we establish a close connection between FI-t-extending modules and FI-t-Baer modules. This is in contrast with [10, Theorem 3.10].

Theorem 3.9. The following statements are equivalent for a module $M$.

(1) $M$ is FI-t-extending.

(2) $M$ is FI-t-Baer and FI-t-cononsingular.

(3) $M$ is FI-t-Baer and $C=t_{M}\left(t_{S}(C)\right)$ for every fully invariant $t$-closed submodule $C$ of $M$.

Proof. (1) $\Rightarrow(2)$. By Theorem 2.2, $M=Z_{2}(M) \bigoplus M^{\prime}$ where $M^{\prime}$ is FIextending. However by [10, Proposition 2.10, Corollary 3.9 and Lemma 3.12], every nonsingular FI-extending module is quasi-Baer, hence $M^{\prime}$ is quasi-Baer. Thus by Theorem 3.2, $M$ is FI-t-Baer. Now we show that $M$ is FI-t-cononsingular. Let $N$ be a fully invariant submodule of $M$ and $K$ be a fully invariant submodule of $N$ such that $t_{S^{\prime}}(K)=t_{S^{\prime}}(N)$ where $S^{\prime}=\operatorname{End}(N)$. By Corollary 2.4(2), $N$ is FI-t-extending. Assume that $C$ is an essential closure of $K+Z_{2}(N)$ 
in $N$. By Theorem 2.2(5), $C$ is a direct summand of $N$, say $N=C \oplus C^{\prime}$. Now if $\pi_{C^{\prime}}: N \rightarrow C^{\prime}$ is the canonical projection, then clearly $\pi_{C^{\prime}} \in t_{S^{\prime}}(K)$, hence $\pi_{C^{\prime}} \in t_{S^{\prime}}(N)$. Thus $C^{\prime}$ is $Z_{2}$-torsion and so $C^{\prime}=0$ (note that $Z_{2}(N) \leq C$ ). Therefore $K+Z_{2}(N) \leq_{e} N=C$. Thus $K \leq_{\text {tes }} N$ by Proposition 1.1(3).

$(2) \Leftarrow(1)$. Since $M$ is FI-t-Baer, $M=Z_{2}(M) \bigoplus M^{\prime}$ where $M^{\prime}$ is quasi-Baer. But $M$ is FI-t-cononsingular, hence $M^{\prime}$ is FI- $\mathcal{K}$-cononsingular by Proposition 3.8(1). Thus by [10, Lemma 3.14], $M^{\prime}$ is FI-extending and so by Theorem 2.2, $M$ is FI-t-extending.

For $(1) \Rightarrow(3)$ one may just follow the proof of $[1$, Theorem $3.9,(1) \Rightarrow(3)]$ by assuming there, that $C$ is a fully invariant t-closed submodule of $M$, and finally $(3) \Rightarrow(1)$ is clear.

\section{FI-t-extending modules and annihilator conditions}

Recall that a module $M$ is quasi-continuous (or $\pi$-injective) if $M$ is an extending module and satisfies condition (C3), that is, if $A$ and $B$ are direct summands of $M$ such that $A \cap B=0$, then $A \oplus B$ is a direct summand of $M$. In [11, Theorem 8], a characterization of a quasi-continuous module relative to an annihilator condition is given: a module $M$ is quasi-continuous if and only if $S=l_{S}(A)+l_{S}(B)$ for any submodules $A$ and $B$ of $M$ with $A \cap B=0$ if and only if $S=l_{S}(A)+l_{S}(B)$ (or equivalently, $S=l_{S}(A) \oplus l_{S}(B)$ ) for any submodules $A$ and $B$ of $M$ which are complements to each other. Analogous to this, a characterization of an extending module relative to an annihilator condition is given in [7, Corollary 2.5]: a module $M$ is extending if and only if for every closed submodule $C$ of $M$ there exists a complement $D$ to $C$ in $M$ such that $S=l_{S}(C)+l_{S}(D)$ (or equivalently, $S=l_{S}(C) \oplus l_{S}(D)$ ). Similar to this, we shall obtain characterizations of an FI-t-extending module relative to an annihilator condition.

Theorem 4.1. The following statements are equivalent for a module $M$ with $S=\operatorname{End}(M)$.

(1) $M$ is FI-t-extending.

(2) For every fully invariant $t$-closed submodule $C$ of $M$ there exists a complement $D$ to $C$ in $M$ such that $S=l_{S}(C) \bigoplus l_{S}(D)$.

(3) For every fully invariant $t$-closed submodule $C$ of $M$ there exists a complement $D$ to $C$ in $M$ such that $S=l_{S}(C)+l_{S}(D)$.

(4) For every fully invariant t-closed submodule $C$ of $M$ there exists a complement $D$ to $C$ in $M$ such that $D+Z_{2}(M)$ is $t$-closed in $M$ and $S=$ $t_{S}(C)+t_{S}(D)$.

Proof. (1) $\Rightarrow(2)$. Let $C$ be a fully invariant t-closed submodule of $M$. By hypothesis $M=C \oplus D$ for some submodule $D$ and so $D$ is a complement to $C$ in $M$. However $C=e M$ and $D=(1-e) M$ for some idempotent $e \in S$, hence $S(1-e)=l_{S}(C)$ and $S e=l_{S}(D)$. Thus $S=l_{S}(C) \bigoplus l_{S}(D)$.

$(2) \Rightarrow(3)$. This is a tautology. 
$(3) \Rightarrow(4)$. By restricting the annihilator condition to fully invariant tclosed submodules in the proof of [7, Lemma 2.1] we deduce that $M=C \oplus D$ and especially $M=Z_{2}(M) \oplus M^{\prime}$ for some submodule $M^{\prime}$ of $M$. Therefore $M=Z_{2}(M) \oplus\left(C \cap M^{\prime}\right) \oplus D$ and so $D \oplus Z_{2}(M)$ is t-closed in $M$. Moreover hypothesis implies that $S=t_{S}(C)+t_{S}(D)$ since $l_{S}(C) \leq t_{S}(C)$ and $l_{S}(D) \leq t_{S}(D)$.

$(4) \Rightarrow(1)$. Let $C$ be a fully invariant t-closed submodule of $M$. By hypothesis $S=t_{S}(C)+t_{S}(D)$ for some complement $D$ to $C$ for which $D+Z_{2}(M)$ is t-closed in $M$. Then $1=\varphi+\psi$ where $\varphi \in t_{S}(C)$ and $\psi \in t_{S}(D)$. Then $C \leq t_{M}(\varphi) \leq t_{M}\left(\varphi^{2}\right)$ and $D \leq t_{M}(\psi) \leq t_{M}\left(\psi^{2}\right)$. Now let $d \in D \cap t_{M}\left(\varphi^{2}\right)$. As $d=\varphi d+\psi d$, we conclude that $\varphi d-\varphi \psi d=\varphi^{2} d \in Z_{2}(M)$ and so $\varphi d \in Z_{2}(M)$ since $\psi \in t_{S}(D)$. Thus $d=\varphi d+\psi d \in Z_{2}(M)$. This implies that $D \cap t_{M}\left(\varphi^{2}\right) \leq$ $Z_{2}(M)$ and so $D \cap t_{M}\left(\varphi^{2}\right)=0$ as $Z_{2}(M) \leq C$ by Proposition 1.2(2). However $D$ is a complement to $C$ in $M$, hence by [8, Corollary 6.23], $C$ is a complement to $D$ in $M$. Thus

$$
C=t_{M}(\varphi)=t_{M}\left(\varphi^{2}\right) .
$$

Similar to the above, we see that $C \cap t_{M}\left(\psi^{2}\right) \leq Z_{2}(M)$ and so $\bar{C} \cap \overline{t_{M}\left(\psi^{2}\right)}=\overline{0}$ where the bar denotes the image in $M / Z_{2}(M)$. It is easy to see that $\bar{C}$ is a complement to $\bar{D}$ in $\bar{M}$. Moreover, $\bar{D}$ is a closed submodule of $\bar{M}$, since $D+Z_{2}(M)$ is t-closed in $M$ by hypothesis. Therefore by [8, Corollary 6.23], $\bar{D}$ is a complement to $\bar{C}$ in $\bar{M}$ and so $\bar{D}=\overline{t_{M}\left(\psi^{2}\right)}$. Hence

$$
D+Z_{2}(M)=t_{M}(\psi)=t_{M}\left(\psi^{2}\right) .
$$

Now we show that $\varphi \psi M \leq Z_{2}(M)$. For this purpose, it suffices to show that $\varphi \psi M \cap(C \bigoplus D) \leq Z_{2}(M)$, since $C \bigoplus D \leq_{\text {tes }} M$. Assume that $\varphi \psi m=c+d$ where $c \in C$ and $d \in D$. From the equality $1=\varphi+\psi$, it is clear that $\varphi \psi=\psi \varphi$. Then $\varphi^{2} \psi^{2} m=\varphi \psi(c+d)=\psi \varphi c+\varphi \psi d \in Z_{2}(M)$ (recall that $\varphi \in t_{S}(C)$ and $\left.\psi \in t_{S}(D)\right)$. Thus $\psi^{2} m \in t_{M}\left(\varphi^{2}\right)=t_{M}(\varphi)$, hence $\psi^{2} \varphi m=\varphi \psi^{2} m \in Z_{2}(M)$. Consequently $\varphi m \in t_{M}\left(\psi^{2}\right)=t_{M}(\psi)$ and so $\varphi \psi m \in Z_{2}(M)$. This implies that $\varphi \psi M \cap(C \bigoplus D) \leq Z_{2}(M)$, as desired.

From $\varphi \psi M \leq Z_{2}(M)$ we conclude that $\psi M \leq t_{M}(\varphi)=C$ and $\varphi M \leq$ $t_{M}(\psi)=D+Z_{2}(M)$. Thus $M=\varphi M+\psi M \leq C \bigoplus D$ and so $C \bigoplus D=M$, that is, $C$ is a direct summand of $M$.

Remark 4.2. In the proof of Theorem 4.1, if we assume that $C$ is an arbitrary t-closed submodule of $M$, then by the same proof, we obtain the following equivalent statements for a t-extending module $M$.

(1) $M$ is t-extending.

(2) For every t-closed submodule $C$ of $M$ there exists a complement $D$ to $C$ in $M$ such that $S=l_{S}(C) \bigoplus l_{S}(D)$.

(3) For every t-closed submodule $C$ of $M$ there exists a complement $D$ to $C$ in $M$ such that $S=l_{S}(C)+l_{S}(D)$.

(4) For every t-closed submodule $C$ of $M$ there exists a complement $D$ to $C$ in $M$ such that $D+Z_{2}(M)$ is t-closed in $M$ and $S=t_{S}(C)+t_{S}(D)$. 
Acknowledgment. The research of the first author was in part supported by a grant from IPM (No. 90130036).

\section{References}

[1] Sh. Asgari and A. Haghany, T-extending modules and t-Baer modules, Comm. Algebra 39 (2011), 1605-1623.

[2] G. F. Birkenmeier, G. Călugăreanu, L. Fuchs, and H. P. Goeters, The fully invariant extending property for abelian groups, Comm. Algebra 29 (2001), no. 2, 673-685.

[3] G. F. Birkenmeier, B. J. Müller, and S. T. Rizvi, Modules in which every fully invariant submodule is essential in a direct summand, Comm. Algebra 30 (2002), no. 3, 13951415.

[4] G. F. Birkenmeier, J. K. Park, and S. T. Rizvi, Modules with fully invariant submodules essential in fully invariant summands, Comm. Algebra 30 (2002), no. 4, 1833-1852.

[5] _ Generalized triangular matrix rings and the fully invariant extending property, Rocky Mountain J. Math. 32 (2002), no. 4, 1299-1319.

[6] _ Modules with FI-extending hulls, Glasg. Math. J. 51 (2009), no. 2, 347-357.

[7] M. A. Kamal and A. M. Menshawy, CS-modules and annihilator conditions, Int. J. Math. Math. Sci. 2003 (2003), no. 50, 3195-3202.

[8] T. Y. Lam, Lectures on Modules and Rings. Graduate Texts in Mathematics, Vol. 189, Springer-Verlag, New York/Berlin, 1998.

[9] A. C. Özcan, A. Harmanci, and P. F. Smith, Duo modules, Glasg. Math. J. 48 (2006), no. 3, 533-545.

[10] S. T. Rizvi and C. S. Roman, Baer and quasi-Baer modules, Comm. Algebra 32 (2004), no. 1, 103-123.

[11] R. Wisbauer, M. F. Yousif, and Y. Zhou, Ikeda-Nakayama modules, Beiträge Algebra Geom. 43 (2002), no. 1, 111-119.

SHADi ASGARI

Department of Mathematical Sciences

ISFAHAN UNIVERSITY OF TECHNOLOGY

ISFAHAN 84156-83111, IRAN

AND SCHOOL OF MATHEMATICS

Institute For RESEARCH In Fundamental Sciences (IPM)

TEHRAN, IRAN

E-mail address: sh_asgari@math.iut.ac.ir

AHMAD HAGHANY

Department of Mathematical Sciences

ISFAHAN UNIVERSITY OF TECHNOLOGY

ISFAHAN 84156-83111, IRAN

E-mail address: aghagh@cc.iut.ac.ir 